\title{
Beware of 'normal' creatine kinase levels in HIV-associated polymyositis
}

\author{
C Kenyon, K Pillay, J M Heckmann
}

To the Editor: Generalised weakness may be a common complaint of persons infected with HIV, but the development of significant proximal weakness requires specific attention. Polymyositis may occur in HIV infection and is readily treatable with prednisone. Elevated creatine kinase (CK) levels have been regarded as an important criterion for diagnosing polymyositis. A study of HIV-associated polymyositis reported similarly elevated CK levels to those observed in non-HIV settings. ${ }^{1}$ However, muscle inflammation can be associated with normal or near-normal CK levels. We report 4 cases of HIV-associated polymyositis in which the diagnosis was almost missed owing to the absence of raised CK levels.

Patient 1 presented with a subacute history of painful proximal muscle weakness and arthralgia (Table I). The CK level was normal, but electromyography (EMG) showed an active myopathic process and a muscle biopsy revealed features of polymyositis. She responded to prednisone but relapsed clinically within 3.5 months of weaning from the steroids. Re-introduction of prednisone $30 \mathrm{mg}$ daily, with subsequent weaning, led to a durable clinical response. Patient 2 presented with a diarrhoeal illness and 2 weeks of proximal muscle weakness. Stool microscopy revealed Cryptosporidium cysts. She was rehydrated and antiretroviral therapy was commenced with resolution of the diarrhoea. Despite adequate rehydration and normal electrolyte levels she remained too weak to walk without assistance. Examination confirmed a proximal myopathy with normal reflexes and normal findings on sensory examination, and EMG and muscle biopsy showed features of polymyositis. Her power improved substantially on prednisone therapy. Patients 3 and 4 both presented with a subacute history of proximal weakness. EMG revealed an active myopathic process in both as well as polymyositis on both muscle biopsies. Patient 3's weakness resolved completely within 2 months on prednisone $60 \mathrm{mg}$. The clinician attending patient 4 assessed the low CK level as suggestive of 'burnt out' myositis and decided against steroid therapy. The patient was commenced on antiretrovirals and within 3 months her power had almost completely normalised.

Division of Infectious Disease and HIV Medicine, Department of Medicine, University of Cape Town

C Kenyon, MB ChB, MPH

Division of Anatomical Pathology, National Health Laboratory Services, Red Cross War Memorial Children's Hospital and University of Cape Town

K Pillay, MB ChB, MMed (Anat Path)

Division of Neurology, Department of Medicine, Groote Schuur Hospital and University of Cape Town

J M Heckmann, MB BCh, PhD

Corresponding author:J M Heckmann (jeanine.heckmann@uct.ac.za)

\section{Discussion}

HIV-associated polymyositis has the same clinical, electromyographic and histological features as polymyositis in HIV-negative subjects. ${ }^{2}$ However, in our 4 patients with HIVassociated polymyositis, normal CK levels delayed the clinical diagnosis of an inflammatory myositis. Although EMG and muscle biopsy showed evidence of polymyositis, we feel that it is important to highlight the importance of a normal CK level in this setting, as many patients may not have access to either EMG or histological examinations. Indeed, in a retrospective review of the clinical and laboratory findings of all the muscle biopsies performed at Groote Schuur Hospital from January 2003 to June 2009, and limiting the analysis to those with clinical probable polymyositis, we found that the CK levels at presentation were considerably lower in the HIV-positive compared with the HIV-negative cohort. As an example, the median CK level in the HIV-positive cohort was less than half that in the HIV-negative cohort (manuscript in preparation).

CK levels relate to the amount of enzyme released by the muscle cells, and their measurement is the most frequently used laboratory test screening for inflammatory myositis. CK levels are usually raised at least 3- to 4-fold in polymyositis. ${ }^{3}$ However, an inflammatory myopathy on muscle biopsy may be associated with a CK level within the normal range, such as in childhood-onset dermatomyositis, ${ }^{4}$ inclusion body myositis and even polymyositis, although the latter accounted for $<15 \%$ of a recent Mayo clinic series. ${ }^{3}$ Interestingly, patients with active rheumatoid arthritis without myositis have lower (by half) CK levels than normal controls, although their muscle aldolase levels are higher than controls. ${ }^{5}$ It has been suggested that an inhibitor, such as enzyme-binding immunoglobulins, could be present in the serum of subjects during systemic inflammation that may falsely 'normalise' the CK level, ${ }^{6}$ and indeed CK levels were found to be reduced after therapeutic administration of intravenous hyperimmune globulin, whereas placebo did not have this effect, irrespective of the autoimmune disease treated. ${ }^{7}$ An alternative explanation for the low CK levels is that a generalised inflammatory state may result in post-transcriptional modification and enhanced degradation of CK. CK is an unstable enzyme and vulnerable to numerous post-transcriptional changes in the setting of generalised inflammation. ${ }^{8}$ The thiol groups of CK are susceptible to oxidation, CK may be glycated, and it can be deactivated by the plasma enzyme carboxypeptidase $\mathrm{N}$ (summarised in Delanghe and De Buyzere ${ }^{8}$ ). Enhanced rates of CK destruction in the setting of infection and inflammation have indeed been shown in vitro. ${ }^{8} \mathrm{HIV}$ infection is characterised by background inflammation resulting in, for example, polyclonal activation of B lymphocytes, ${ }^{9}$ and these effects on CK activity have not been investigated.

Polymyositis is the most common HIV-associated muscle disease, although in rare cases uncomplicated myalgias, 
Table I. Clinical and laboratory characteristics of 4 patients with HIV-associated polymyositis with normal or near-normal creatine kinase levels

\begin{tabular}{|c|c|c|c|c|}
\hline & Patient 1 & Patient 2 & Patient 3 & Patient 4 \\
\hline Age (yrs), gender & $29, \mathrm{~F}$ & $39, \mathrm{~F}$ & $35, \mathrm{~F}$ & $22, \mathrm{~F}$ \\
\hline Duration of symptoms (mo.) & 3 & 0.5 & 2 & 7 \\
\hline Concomitant medications & Nil & Co-trimoxazole & Nil & Nil \\
\hline Antiretrovirals at onset of weakness & No & No & No & No \\
\hline Shoulder abduction (MRC grade)* & $4-$ & $4-$ & $4-$ & 3 \\
\hline Hip flexion power (MRC grade)* & 2 & $4-$ & $4-$ & 3 \\
\hline Potassium (mmol/1) (N 3.3 - 3.5) & $\mathrm{N}$ & 3.3 & $\mathrm{~N}$ & $\mathrm{~N}$ \\
\hline TSH & $\mathrm{N}$ & $\mathrm{N}$ & $\mathrm{N}$ & $\mathrm{N}$ \\
\hline CD4+ count, cells $/ \mu \mathrm{l}$ & 389 & 10 & 83 & 190 \\
\hline Initial CK levels (IU/1) (N 26 - 140) & 91 & 102 & 89 & 199 \\
\hline
\end{tabular}

asymptomatic elevation of CK and rhabdomyolysis may also be encountered. ${ }^{2}$ In a series of HIV-associated polymyositis, 10 of 13 cases developed symptoms on ART, ${ }^{1}$ whereas all 4 of our patients were ART naïve. The majority of those patients, much like ours, responded to prednisone treatment. The decision to treat with immunosuppressives in the setting of HIV infection involves a complex balancing act to try to regain control of the HIV-induced immune dysregulation. Indeed, in patient 4 the beneficial effect of ART appeared to be sufficient to regain control of the auto-immune process.

We highlight the fact that a patient with HIV-associated polymyositis may have normal serum CK levels, and in this setting one should perform an EMG and/or a muscle biopsy or at least assess other muscle enzyme levels such as serum aldolase and myoglobin. ${ }^{6}$

\section{References}

1. Johnson RW, Williams FM, Kazi S, Dimachkie MM, Reveille JD. Human immunodeficiency virus-associated polymyositis: a longitudinal study of outcome. Arthritis Rheum 2003; 49(2): 172-178.

2. Robinson-Papp J, Simpson DM. Neuromuscular diseases associated with HIV-1 infection. Muscle Nerve 2009; 40: 1043-1053.

3. Chahin N, Engel AG. Correlation of muscle biopsy, clinical course, and outcome in PM and sporadic IBM. Neurology 2008; 70(6): 418-424

4. Peloro TM, Miller OF 3rd, Hahn TF, Newman ED. Juvenile dermatomyositis: a retrospective review of a 30-year experience. J Am Acad Dermatol 2001; 45(1): 28-34.

5. Sanmarti R, Collado A, Gratacos J, et al. Reduced activity of serum creatine kinase in rheumatoid arthritis: a phenomenon linked to the inflammatory response. Br J Rheumatol 1994; 33(3): 231-234.

6. Rosalki SB. Low serum creatine kinase activity. Clin Chem 1998; 44(5): 905.

7. Koffman BM, Dalakas MC. Effect of high-dose intravenous immunoglobulin on serum chemistry, hematology, and lymphocyte subpopulations: assessments based on controlled 8. Delanghe JR, De Buyzere ML. Low creatine kinase activity in rheumatoid arthritis. J Rheumatol 1997; 24: 231-232.

9. Russo S, Lopalco L. Is autoimmunity a component of natural immunity to HIV? Curr HIV Res 2006; 4(2): 177-190.

Accepted 26 November 2009. 